\title{
Effects of combined growth hormone and testosterone treatments in a rat model of micropenis
}

\author{
Jin Kyu Oh', Young Jae Im², Kwanjin Park² and Jae-Seung Paick ${ }^{2}$ \\ ${ }^{1}$ Department of Urology, College of Medicine, Gachon University, Incheon, Republic of Korea \\ ${ }^{2}$ Department of Urology, College of Medicine, Seoul National University, Seoul, Republic of Korea \\ Correspondence should be addressed to K Park: urodori@naver.com
}

\begin{abstract}
Although it is well known that penile growth is dependent on androgens, few clinical studies have reported successful treatment of micropenis with testosterone, likely due to concerns regarding the efficacy and safety of prolonged testosterone use. Thus, we assessed the synergenic effects of growth hormone (GH) treatments with and without testosterone on phallic growth in a rat model of micropenis. Fifty Sprague-Dawley rats were assigned to control (C), microphallus (MP), testosterone, GH (G) and GH plus testosterone (GT) treatment groups, and microphallus was induced by secondary hypogonadism. Pre-pubertal treatments with testosterone, $\mathrm{GH}$ or the combination were initiated from 7 days after birth and were maintained until 12 weeks of age. To assess the efficacy of treatments, phallic dimensions were determined and histological markers of cavernosal integrity were evaluated. Skeletal and gonadal safety profiles of the treatments were then assessed according to right tibial lengths and testicular weights, respectively. No monotreatments normalised penile dimensions, whereas combination treatments led to complete restoration. The combination treatment also prevented decreases in histological indicators of cavernosal integrity, including smooth muscle actin and collagen III expression levels and fat globule accumulation and sinusoidal density. These synergenic effects of GH treatments on penile growth may follow changes in androgen receptor expression levels and were accompanied by decreased testicular volume losses. Although the physiological conditions of phallic growth differ between humans and rats, this proof-of-concept study provides a strategy for circumventing the problems of testosterone monotherapy for human micropenis.
\end{abstract}

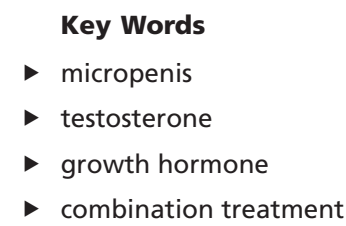

Endocrine Connections (2018) 7, 1150-1157

\section{Introduction}

The term micropenis encompasses a range of congenital and acquired conditions that present as abnormally short penises with a stretched length of more than 2.5 standard deviations (s.D.) below the mean for a particular age, albeit with no hypospadias, as indicated by normal morphology of the urethral meatus at the tip of the glans penis. Micropenis is commonly associated with testosterone deficiencies from 12 weeks of gestation, with normal placental human chorionic gonadotropininduced testosterone levels during organogenesis (1). Among patients with hypogonadotropic hypogonadism, functional deficiencies are more frequently associated with foetal luteinising hormone than with disorders of sexual differentiation. Less frequent causes include growth hormone $(\mathrm{GH})$ deficiencies and idiopathic functional abnormalities of the hypothalamic-pituitary-testicular
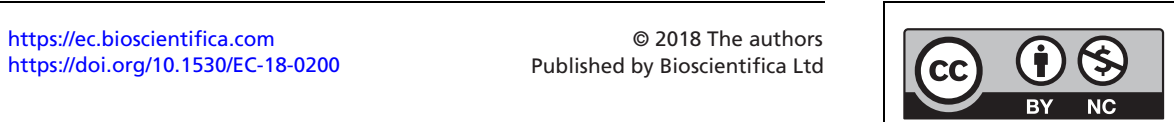

This work is licensed under a Creative Commons Attribution-NonCommercial 4.0 International License. 
axis, and overt abnormalities are generally absent under these conditions $(2,3)$.

Patients with micropenis are often dissatisfied with their sexual quality of life and cosmetic appearance and stigmatisation is common $(4,5)$. Hence, hormone treatments have been considered, and these reportedly result in longer penile lengths when administered early $(6,7)$, albeit with concerning side effects. In a study of hypogonadotropic microphallic rats by Husmann et al. (8), testosterone treatments increased pre-pubertal penile lengths but resulted in smaller phallic sizes than in normal rats, suggesting that early administration leads to premature termination of pubertal phallic growth. These observations were associated decreased androgen receptor (AR) expression levels before puberty, clearly due to the presence of exogenous testosterone. Moreover, effects on the growth of testicular germ cells and bone may warrant safety concerns, and these remain insufficiently characterised.

GH deficiency is the primary cause of micropenis, even in the presence of intact hypothalamic-pituitarygonadal axis (9). Although the underlying molecular mechanisms have not been described, the GH-insulinlike growth factor 1 (IGF1) axis is a proactive mediator of the actions of testosterone and its potent derivative dihydrotestosterone. Accordingly, stimulation of IGF1 signalling prevented reductions of AR expression and enhanced penile fibroblast proliferation and testis growth, indicating a target for the treatment of micropenis (10). Hence, under conditions of normal GH and IGF1 activities, penile dimensions may be increased without the side effects of conventional androgen treatments.

Herein, we characterised the effects of $\mathrm{GH}$ monotherapy and GH-testosterone combination therapy on phallic dimensions, structural integrity, bone growth and testicular size in microphallic rats.

\section{Materials and methods}

\section{Animals and experimental protocol}

All animal procedures were reviewed and approved by the Institutional Animal Care and Use Committee of Seoul National University. Animal husbandry was performed in compliance with the Association for Assessment and Accreditation of Laboratory Animal Care and the National Institutes of Health guidelines. Seven timedpregnant Sprague-Dawley rats were obtained at 1 week before parturition, and after parturition, pups were raised with their mothers until 2 weeks of age. Male pups were distinguished from females by longer anogenital lengths on the third postnatal day, although this distinction was not always clear, leading to differing numbers of pups between study groups. Pups were assigned to the following treatment groups: normal control (C), untreated microphallic rats (MP), microphallic rats treated with GH only $(G)$, microphallic rats treated with testosterone and microphallic rats treated with both $\mathrm{GH}$ and testosterone (GT).

Microphallus was induced in rat pups by weekly administration of leuprolide acetate (74381536, intramuscular administration of $5 \mu \mathrm{g} / \mathrm{kg}$ in $0.1 \mathrm{~mL}$ saline solution, Sigma-Aldrich) at $0.1-0.4 \mathrm{mg} / \mathrm{kg}$, with biweekly dose increases from postpartum day 5 . From postpartum day 7, testosterone enanthate (Savient Pharmaceuticals, East Brunswick, NJ, USA) was administered intramuscularly at $0.4 \mathrm{mg} / \mathrm{kg} /$ week. GH (recombinant human growth hormone bulk solution, LG Life Sciences, Korea) treatments were administered subcutaneously on alternate days at $2.5 \mathrm{mg} / \mathrm{kg}$, and after 14 weeks, rats were killed by aspiration of blood from the heart. Following anthropometric measurements of body weights and phallic dimensions, penises were excised at the level of the crus and were examined under stereomicroscopic control. To evaluate the effects of treatments on bone growth, right tibia lengths were measured using a digital calliper with an accuracy of $0.01 \mathrm{~mm}$ (Merox, Vienna, Austria). The area below the penile os (distal area) that shows the typical bilobular shape of the corpus cavernosum was isolated, sliced and fixed in $4 \%$ formalin for immunohistochemical analyses. Western blotting analyses were performed using tissue samples from the area near the penile crus (proximal side), which includes smooth muscle. These samples were resected, snap-frozen in liquid nitrogen and stored at $-80^{\circ} \mathrm{C}$.

\section{Penile anthropometry}

Body weights were measured every 2 weeks and at the time of necropsy. Stretched penile lengths were recorded by stretching the phallus and measuring the length from the palpable proximal penile tip to the tip of the phallus using micro-callipers. Adjusted penile lengths were recorded as ratios of penile and tibial lengths.

\section{Plasma testosterone and IGF1 assays}

Aliquots of blood were centrifuged, and the resulting plasma samples were stored at $-20^{\circ} \mathrm{C}$. After thawing

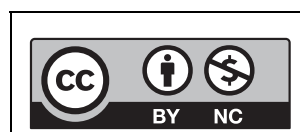

This work is licensed under a Creative Commons Attribution-NonCommercial 4.0 International License. 
plasma, assays of testosterone and IGF1 were performed using commercial ELISA kits (Abcam and R\&D Systems, respectively). All assays were conducted in duplicate, and mean values were calculated. Assays were sensitive to $0.07 \mathrm{ng} / \mathrm{mL}$ testosterone and $4.4 \mathrm{pg} / \mathrm{mL}$ IGF1, with reported inter-assay coefficients of variation of 5 and $4.6 \%$, respectively.

\section{AR assays}

Proximal parts of penises were homogenised individually in a buffer containing $10 \mathrm{mM}$ Tris (pH 7.4), $1.5 \mathrm{mM}$ EDTA, $0.1 \%$ glycerol, $1 \mathrm{mM}$ 2-mercapto-ethanol and $10 \mathrm{mM} \mathrm{Na}$ molybdate. Homogenates were centrifuged at $150,000 \boldsymbol{g}$ for $30 \mathrm{~min}$, and cytosolic fractions were collected. Aliquots of the penile cytosol fractions containing $120 \mu \mathrm{g}$ of protein were run on $7.5 \%$ polyacrylamide (PAGE) gels in sodium dodecyl sulphate, and the proteins were then transferred to PVDF membranes $(0.45 \mu \mathrm{m}$, GE Healthcare Bio-Sciences) at $100 \mathrm{~V}$ for $50 \mathrm{~min}$. Membranes were blocked with $5 \%$ bovine serum albumin in PBS solution for $1 \mathrm{~h}$ and were then incubated with primary rabbit polyclonal antibody against a $110 \mathrm{kDa}$ rat AR protein from the HepG2 nuclear region (1:1000, ab133273, Abcam). Subsequently, membranes were rinsed briefly with TBS-T buffer and were incubated with secondary horseradish peroxidase-conjugated antibody (1:3000) for $60 \mathrm{~min}$. Bands were detected using enhanced chemiluminescence (GE Healthcare Bio-Sciences), and exposed films were scanned for densitometric analyses of band densities.

\section{Histomorphometry}

Fixed specimens were processed and embedded in paraffin wax and were then sectioned and stained with haematoxylin and eosin. Slides were de-paraffinised using three washes with xylene and were then rehydrated by incubating in a graded series of alcohol (100, 95 and 70\%) and water solutions for $5 \mathrm{~min}$. Unless otherwise stated, all washes were performed three times in PBS containing $0.05 \%$ Tween ( $\mathrm{pH} 7.4$ ) for $5 \mathrm{~min}$ each, and all incubation steps were conducted in a humid chamber at room temperature. Antigen retrieval was achieved by heating slides in a microwave oven in $0.01 \mathrm{M}$ sodium citrate ( $\mathrm{pH}$ 6.0), cooling for $30 \mathrm{~min}$ and then washing 3 times in PBS containing 0.05\% Tween. Endogenous peroxidase activity was blocked using 1\% hydrogen peroxide in methanol for $30 \mathrm{~min}$, and non-specific binding was blocked by incubating in normal horse serum for $1 \mathrm{~h}$ (Vector Laboratories, Inc.). Slides were incubated with an anti-ACTA2 primary antibody (1:1000, sc-130617, Santa Cruz Biotechnology) and collagen types I (1:1000, ab34710, Abcam) and III (1:1000, ab7778, Abcam) for $1.5 \mathrm{~h}$ at room temperature. After washing, an avidin-biotin-HRP complex (VECTASTAIN Elite ABC kit, Vector Labs) was prepared and added according to the manufacturer's instructions. Finally, 3,3,0-diaminobenzidine was used as the chromogen substrate and photomicrographs were taken using an Olympus BX73 microscope (Olympus) under bright field illumination.

Relative expression levels of smooth muscle actin (SMA) and collagen types I and III were determined, and numbers of fat globules and sinusoid densities were calculated from photomicrographs taken at $100 \times$ magnification using Image Pro Plus software (version 4.5.0.29z, Media Cybernetics, Rockville, MD, USA). All variables were assessed in two different areas of the corpus cavernosum for each animal. Areas with SMA or collagen expression were calculated from percentages of pixels with the same colour (brown for SMA and deep blue for collagen) using a histogram tool after colour segmentation of the image as described previously (11). Similarly, sinusoidal densities were calculated by dividing the sum of sinusoids by the total area of the trabecular network. Numbers of fat globules were counted and averaged directly.

\section{Statistical analysis}

Data are expressed as means \pm s.D. Data were analysed using GraphPad Prism version 6 (Graph Pad Software Inc.), and differences were identified using one-way ANOVA followed by the Bonferroni post hoc test.

\section{Results}

\section{Experimental overview, anthropometry and plasma} testosterone and IGF1 levels

Pregnant female rats predominantly gave birth to 8-14 pups at a time, and 3-11 of these were males and were allocated to treatment groups. A total of 37 male rats were allocated to groups C, MP, G, testosterone and GT $(6,8$, 9,6 and 8 rats, respectively). Anthropometric assessments of mean body weights, right tibia lengths and testicular weights are presented with plasma testosterone and IGF1 levels in Table 1. After treatments, mean body weights and tibia lengths were comparable between all experimental groups. In contrast, leuprolide pretreatment caused

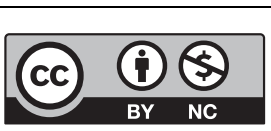

This work is licensed under a Creative Commons Attribution-NonCommercial 4.0 International License. 
Table 1 Comparison of mean body weight, mean testicular weight, mean plasma testosterone and mean IGF-1 among experimental groups.

\begin{tabular}{|c|c|c|}
\hline & $\mathbf{C}$ & MP \\
\hline Body weight (g, s.D.) & $588(34)$ & $579(28)$ \\
\hline Testis volume (mL, s.D.) & $1.36(0.11)$ & $0.97(0.05)^{b}$ \\
\hline Tibial length (mm) & $38.4(2.6)$ & $36.6(3.1)$ \\
\hline $\begin{array}{l}\text { Plasma testosterone (ng/mL, } \\
\text { S.D.) }\end{array}$ & $3.72(1.91)^{a}$ & $0.44(0.05)^{b}$ \\
\hline Plasma IGF-1 (ng/mL, S.D.) & $1445(155)$ & $1432(232)$ \\
\hline
\end{tabular}

\begin{tabular}{c}
\hline $\mathbf{G}$ \\
\hline $575(39)$ \\
$1.26(0.08)^{\mathrm{a}}$ \\
$39.1(2.2)$ \\
$2.08(0.51)^{\mathrm{a}}$
\end{tabular}

\begin{tabular}{c}
\hline Testosterone \\
\hline $552(44)$ \\
$0.83(0.06)^{\mathrm{ab}}$ \\
$35.5(3.3)$ \\
$27.5^{\mathrm{a}, \mathrm{b}}$
\end{tabular}

\begin{tabular}{c}
\hline $\mathbf{G T}$ \\
\hline $567(45)$ \\
$1.10(0.09)^{\mathrm{a}, \mathrm{b}}$ \\
$36.8(2.9)$ \\
$29.2^{\mathrm{a}, \mathrm{b}}$
\end{tabular}

secondary hypogonadism, with significant reductions in testicle volumes, and these conditions were further aggravated by testosterone treatments in MP and G groups. Treatments with GH led to significantly larger testis volume and higher plasma testosterone level, ameliorating changes associated with hypogonadism, but did not correct the problem completely. Compared with rats in the $\mathrm{C}$ group, those in the MP group had significantly lower mean plasma testosterone levels. Treatments with testosterone and $\mathrm{GH}$ resulted in corresponding increases in plasma levels of these agents, whereas plasma testosterone levels were slightly but significantly higher in the G group than in the MP group. Plasma IGF1 levels were lower in testosterone-treated rats that in untreated rats, and mean plasma IGF1 concentrations in the GT group did not differ significantly with those in the $\mathrm{C}$ group, despite GH supplementation.

\section{Penile anthropometry}

Comparisons of phallic lengths and girths are presented in Fig. 1. Microphallus was confirmed in rats of the MP group, with significantly smaller mean phallic dimensions than in the $\mathrm{C}$ group. Phallic dimensions in the $\mathrm{G}$ group did not differ significantly with those in the MP group, and whereas those in the testosterone group were significantly improved, they were not restored to control dimensions. In contrast, combination treatments normalised phallic dimensions to $\mathrm{C}$ sizes. To assess bone growth, adjusted penile lengths were calculated as the ratio of penile and right tibial lengths. Differences in this parameter were similar to those of penile lengths, indicating minimal effects of the present treatments on bone growth.

\section{Androgen receptor expression in penile tissues}

Comparisons of penile (Fig. 2) AR expression levels in 14-week-old rats revealed higher expression in the MP group than that in the $\mathrm{C}$ group. Treatments with

$\begin{array}{lr}\text { https://ec.bioscientifica.com } & \odot 2018 \text { The authors } \\ \text { https://doi.org/10.1530/EC-18-0200 } & \text { Published by Bioscientifica Ltd }\end{array}$

testosterone alone suppressed AR expression, whereas treatments with $\mathrm{GH}$ resulted in increased expression of AR. The highest AR expression levels were observed in the G group, and these were comparable MP and GT groups.
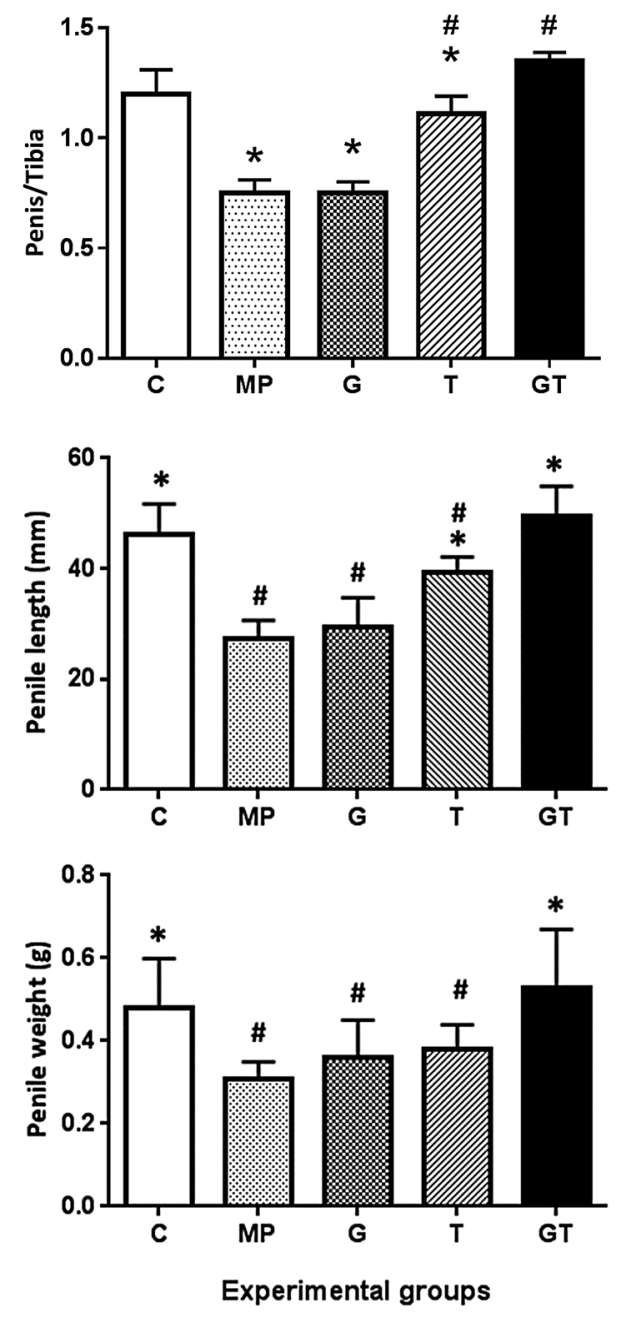

Figure 1

Comparisons of stretched penile lengths, penile weights and penile length/ right tibial length ratios among the five study groups; comparisons of treatments are made with control (C) and microphallus (MP) groups; $* P<0.05$ compared with the MP group; ${ }^{\#} P<0.05$ compared with the $C$ group.

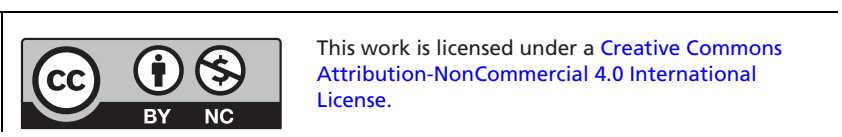



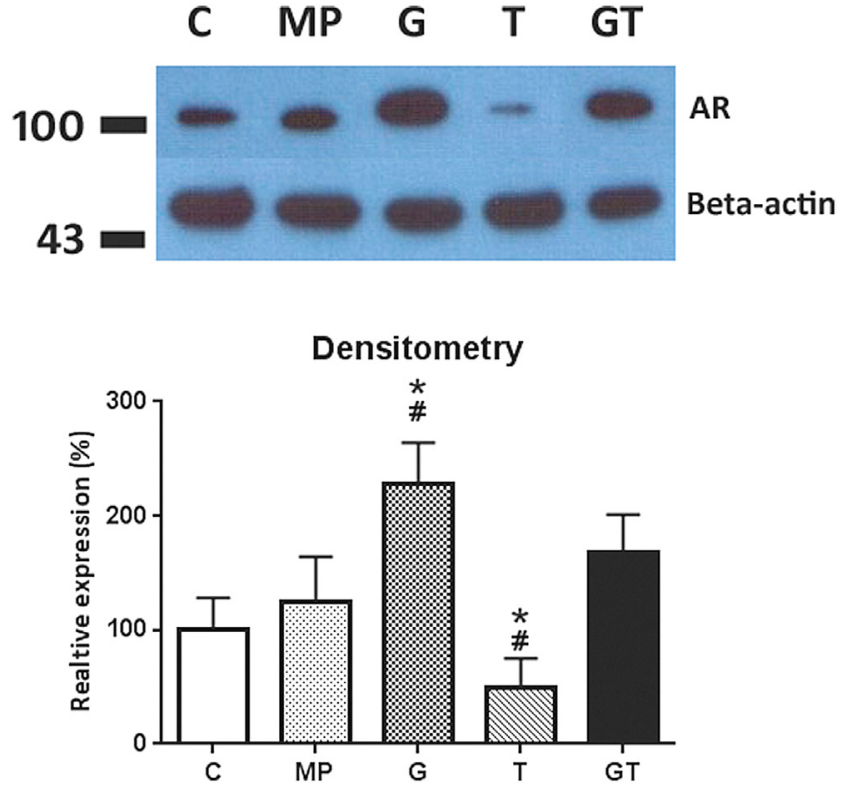

Figure 2

Comparisons of penile androgen receptor (AR) expression between treatment groups; representative traces and corresponding densitometric analyses are presented in upper and lower panels, respectively. Comparisons were made with $C$ and MP groups; ${ }^{*} P<0.05$ compared with the MP group; ${ }^{*} P<0.05$ compared with the $C$ group.

\section{Histomorphometry}

Exposures to hypogonadal insults after birth led to several obvious histologic changes (Fig. 3A and B). Compared with rats of the $\mathrm{C}$ group, MP rats had reduced SMA expression control and increased collagen I and III expression levels. Both collagen types accumulated densely throughout the trabeculae. Moreover, multiple fat globules were found around sinusoidal spaces, and decreased sinusoidal densities were identified. Treatments with GH significantly reduced the expression of collagen III, but did not affect other histologic parameters. Moreover, testosterone treatments significantly increased SMA expression levels and sinusoidal densities and led to marked decreases in fat globule numbers. Expression levels of collagens I and III, however, were not influenced by testosterone treatments alone.

All histologic parameters were improved by combination therapy, with comparable SMA expression levels, numbers of fat globules and sinusoidal densities as those in the $\mathrm{C}$ group. But collagen III levels were only partially restored, and no changes in collagen I levels were identified.

\section{Discussion}

After inducing hypogonadotropic hypogonadism in microphallic rats, significantly smaller phalli were accompanied by reductions in smooth muscle and cavernosal spaces and increased collagen levels and fat globule accumulations. Treatments with GH alone did not affect phallic dimensions, and only partial improvements of collagen III levels were observed under these conditions. Treatments with testosterone alone led to improvements in phallic dimensions and other histologic features, but failed to restore these features to controlled levels. In contrast, combination treatments completely normalised all penile dimensions. Histological assessments showed corresponding benefits of combined hormone treatments, and although collagen I overexpression was not prevented, all treated microphallic rats had significantly decreased testicular volumes, and further losses of testicular volumes due to testosterone monotherapy were ameliorated.

The salient observation of this study was that cotreatments with GH augment the tropic effects of testosterone. We confirmed that testosterone monotherapy significantly, albeit partially, restores penile dimensions in hypogonadal rats, as shown previously $(12,13)$. Our data also corroborate previous observations of the limited tropic effects of GH monotherapy in the absence of GH deficiencies (14). In contrast, the tropic effects of combined treatments with GH and testosterone were sufficient to normalise penile dimensions. Because GH treatments enhanced AR expression, we suggest that the effects of cotreatments with testosterone are mediated by this receptor and that the ensuing signalling normalises phallic growth. Hence, the suboptimal preclinical results of testosterone treatments may reflect insufficient AR expression.

We observed abnormal structural integrity under the present conditions of microphallus. Previous reports show no relationship between penile lengths and sexual satisfaction (15), whereas other studies indicate that patients with micropenis have unsatisfactory or no sexual intercourse for reasons that were not specified (4). Taken together, these studies suggest that although psychological factors likely contribute to the sexual insufficiencies of hypogonadism, penile structural features are at least partly responsible. In accordance, Traish et al. (16) reported loss of smooth muscle and accumulation of adipocytes in hypogonadal rats, and these pathological traits were adequately addressed by testosterone treatments alone. In addition to confirming these observations, we found changes in collagen expression and sinusoidal densities, suggesting that micropenis is compounded by structural integrity and that combination treatments with testosterone and GH restore penis sizes and structural integrity.

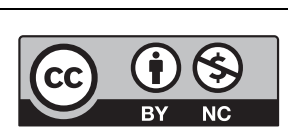

This work is licensed under a Creative Commons Attribution-NonCommercial 4.0 International License. 
3A

Collagen 1
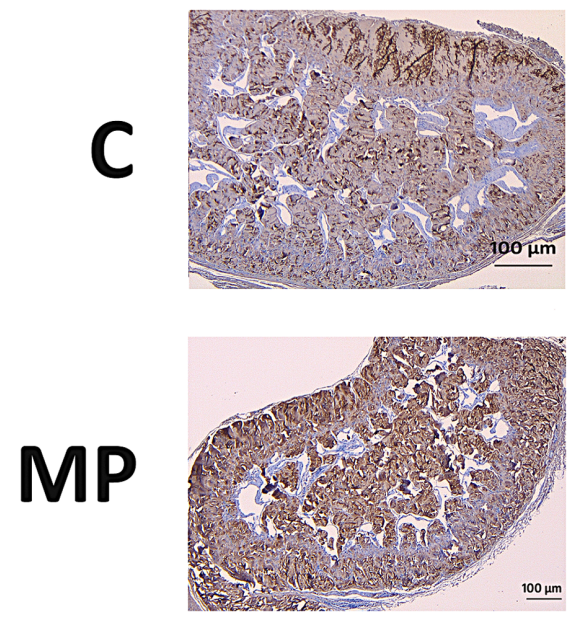

G
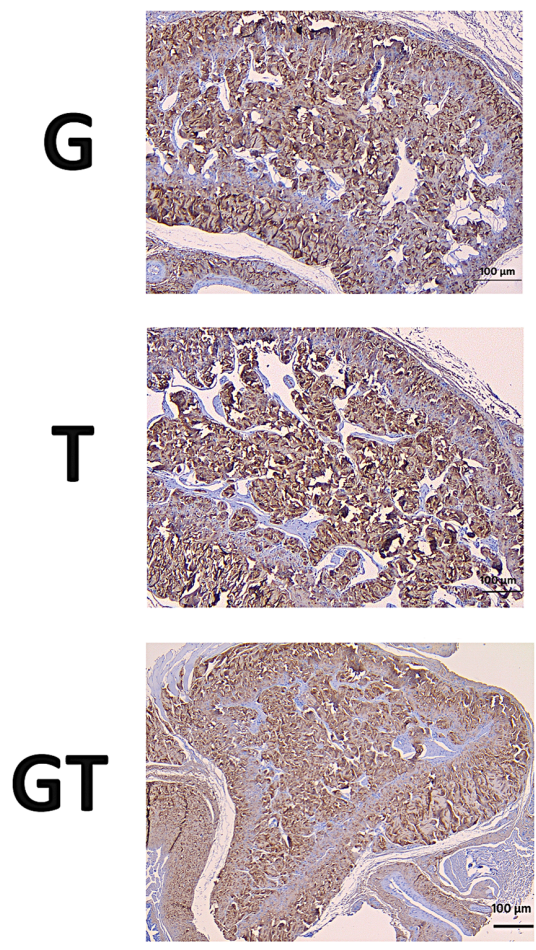

Collagen 3
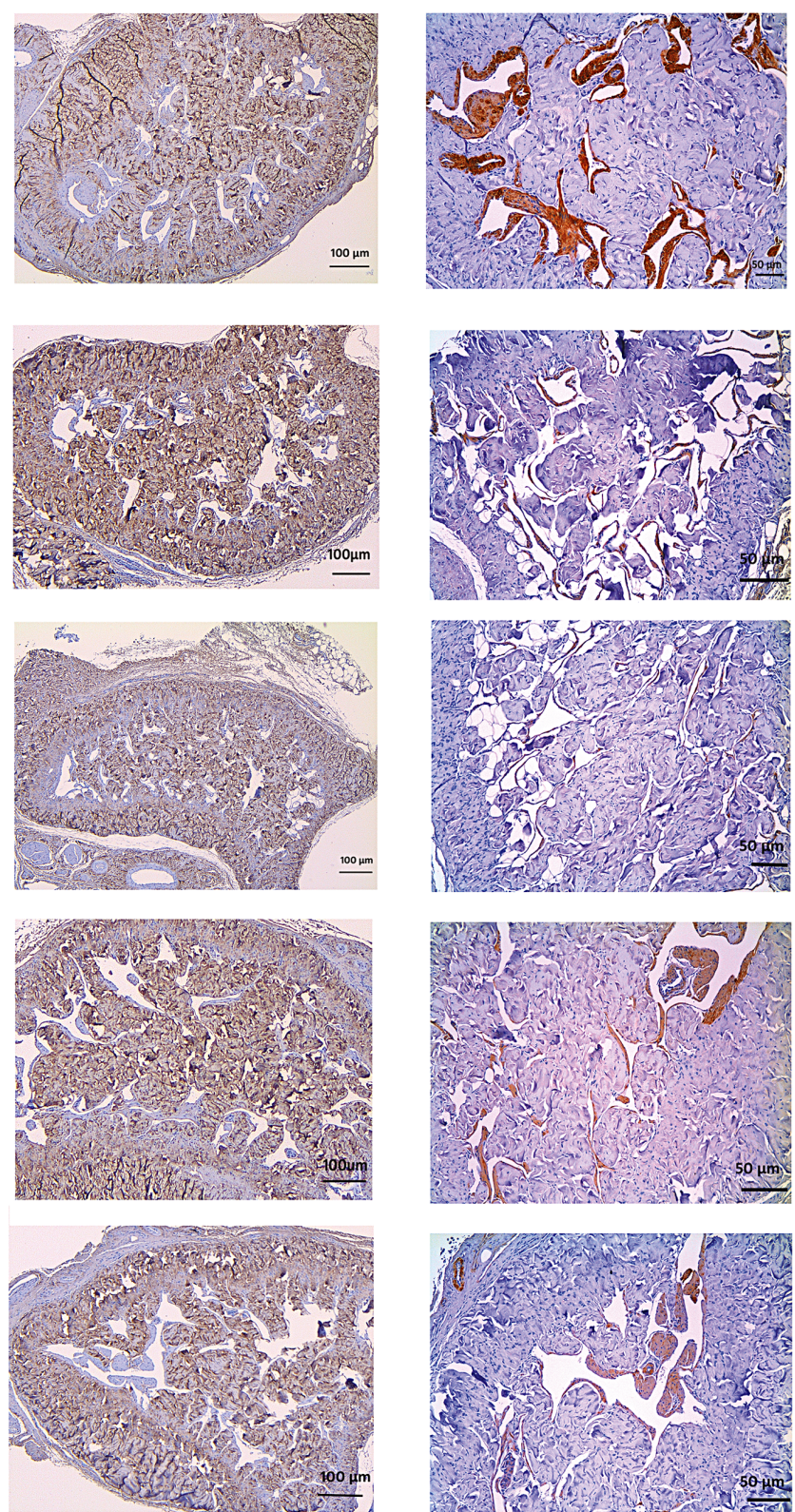

SMA
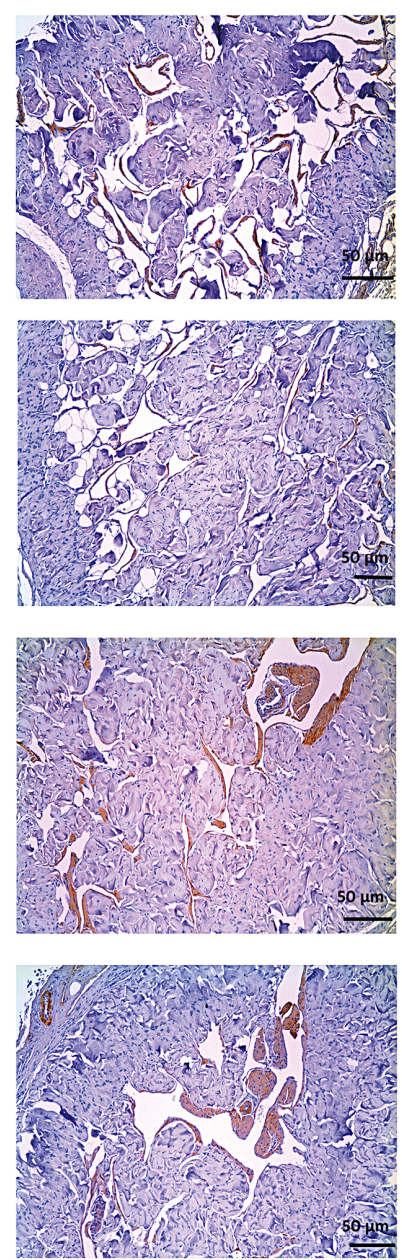

3B
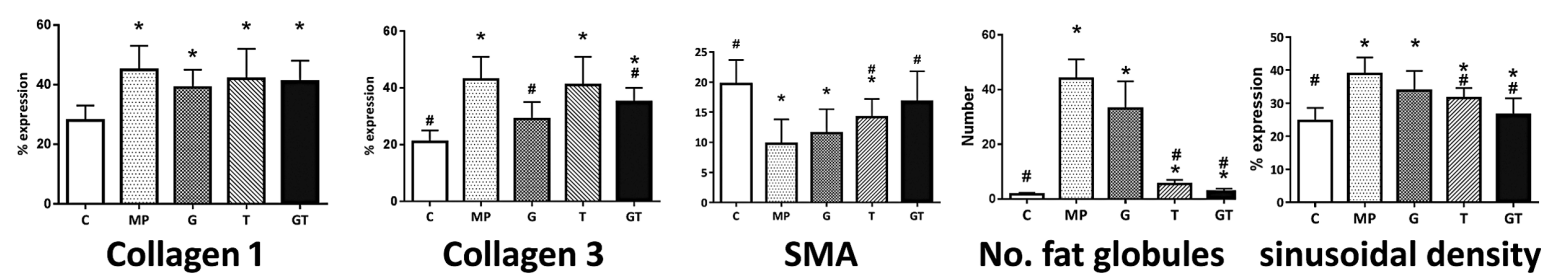

Figure 3

(A) Immunohistochemical localisation of collagens I and III and smooth muscle actin; relative expression levels were determined semiquantitatively; scale bar, $100 \mu \mathrm{m}$. (B) Relative expression levels of collagens I and III and sinusoidal densities and the numbers of fat globules; expression data are presented in the densitogram. Comparisons were made with $C$ and MP groups; ${ }^{*} P<0.05$, compared with the MP group; ${ }^{\#}<0.05$ compared with the $C$ group.

https://ec.bioscientifica.com

https://doi.org/10.1530/EC-18-0200 (c) 2018 The authors Published by Bioscientifica Ltd

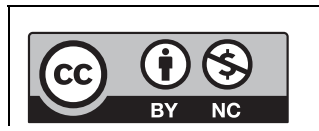

This work is licensed under a Creative Commons Attribution-NonCommercial 4.0 International License. 
Although collagen is a major constituent of extracellular matrixes and is an essential component of the penis, excessive extracellular matrix is undesirable and collagen accumulation may hinder sinusoidal engorgement, leading to reduced sinusoidal densities. Collagen accumulation in patients with hypogonadism is often referred to as penile fibrosis (17) and may prohibit penile growth and contribute to venogenic erectile dysfunction and potentially reduced sexual activity. In our experiments, collagen III overexpression was alleviated by $\mathrm{GH}$ treatments. Although collagen I expression persisted under these conditions, these benefits are exclusive to $\mathrm{GH}$ treatments, supporting its use in combination with testosterone to limit penile fibrosis and preserve structural integrity. Although the implications of increased collagen III levels on penile function require further study, neither of the present monotreatments were ineffective at reducing collagen III levels, despite failing to affect penile dimensions.

Adverse effects of neonatal hypogonadism on subsequent corporal integrity were previously investigated by Okumu et al. (17). In their study, neonatal androgen deficiencies were associated with reduced expression levels of genes and proteins that play roles in smooth muscle differentiation and tone. Taken with these studies, our data indicate that proactive treatments of micropenis may successfully preserve penile integrity and penile dimensions. Specifically, the side effects of prolonged androgen exposure may be alleviated by cotreatments with GH.

We found that GH treatments alone or in combination prevented loss of testicular function beyond that due to hypogonadism. Although testis volumes in the MP group were almost 30\% smaller than in control rats, they were significantly larger in hypogonadal rats treated with GH. Conversely, rats receiving testosterone only suffered greater losses of testis volumes than rats in the MP group. Although the mechanisms behind the tropic effects of $\mathrm{GH}$ on testis are yet to be clarified, several clinical (18) and preclinical reports (19) suggest that GH treatments facilitate testicular growth. Our data are in agreement with studies showing that serum testosterone levels are increased by GH treatments, although testosterone treatments decreased serum IGF1 expression, potentially diminishing the associated benefits. These observations further support the use of $\mathrm{GH}$ as a treatment for micropenis that ameliorates the adverse effects of testosterone monotherapy on testicle growth.

Although changes in tibial lengths corresponded with changes in ratios of penile and tibial lengths, prolonged treatments with testosterone might affect bone growth. Oestrogen rather than testosterone, however, played a major role in bone metabolism and retarded bone growth when present in excess (20). Because the present rats had induced hypogonadism, anti-osteogenic effects of oestrogen are unlikely to reflect limited aromatisation of testosterone. Although high testosterone levels were observed in rats receiving exogenous testosterone, these were not sustained following administration of injectable testosterone in other studies. Thus, in the present rats, the lack of aberrant bone growth may be explained by hypogonadism and the temporary actions of exogenous testosterone. Yet, in previous studies, exogenous testosterone affected bone growth in eugonadal subjects, suggesting that assessments of tibial lengths are poorly representative of bone growth and may be insufficient to confirm the osteopathic safety of testosterone treatments. Assessing tibial bone mass might reveal the difference that was not seen in mere comparison of length. These questions will be subjects of future studies of more factors in relation to bone metabolism.

Notwithstanding the limitations of our assessments of safety, our experiments suggest that combination therapy with testosterone and GH can circumvent the problems associated with monotherapies for hypognadism. In particular, as an adjunct to testosterone, GH may enhance penile growth and preserve structural integrity.

\section{Declaration of interest}

The authors declare that there is no conflict of interest that could be perceived as prejudicing the impartiality of the research reported.

\section{Funding}

This study was supported by grant $04-2010-0780$ from the SNUH research fund.

\section{Author contribution statement}

Conception and design were conducted by Jin Kyu Oh, Kwanjin Park. Acquisition of data was done by Jin Kyu Oh, Young Jae Im. Analysis and interpretation of data were conducted by Young Jae Im, Kwanjin Park. Drafting the article was by Jin Kyu Oh, Young Jae Im. Revising it for intellectual content was by Jae Seung Paick, Kwanjin Park. Final approval of the completed article was by Jin Kyu Oh, Young Jae Im, Jae Seung Paick, Kwanjin Park.

\section{References}

1 Grinspon RP, Loreti N, Braslavsky D, Valeri C, Schteingart H, Ballerini MG, Bedecarras P, Ambao V, Gottlieb S, Ropelato MG, et al. Spreading the clinical window for diagnosing fetal-onset hypogonadism in boys. Frontiers in Endocrinology 20145 51. (https:// doi.org/10.3389/fendo.2014.00051)

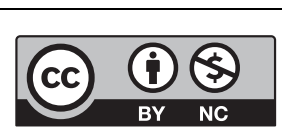

This work is licensed under a Creative Commons Attribution-NonCommercial 4.0 International License. 
2 Husmann DA. Micropenis: an animal model and its human correlates. Advances in Experimental Medicine and Biology 2002511 41-54; discussion 5446.

3 Wiygul J \& Palmer LS. Micropenis. Scientific World Journal 201111 1462-1469. (https://doi.org/10.1100/tsw.2011.135)

4 Callens N, De Cuypere G, Van Hoecke E, T'Sjoen G, Monstrey S, Cools M \& Hoebeke P. Sexual quality of life after hormonal and surgical treatment, including phalloplasty, in men with micropenis: a review. Journal of Sexual Medicine 201310 2890-2903. (https://doi. org/10.1111/jsm.12298)

5 Ciancio F, Lo Russo G, Innocenti A, Portincasa A, Parisi D \& Mondaini N. Penile length is a very important factor for cosmesis, function and psychosexual development in patients affected by hypospadias: results from a long-term longitudinal cohort study. International Journal of Immunopathology and Pharmacology 201528 421-425. (https://doi.org/10.1177/0394632015576857)

6 Bin-Abbas B, Conte FA, Grumbach MM \& Kaplan SL. Congenital hypogonadotropic hypogonadism and micropenis: effect of testosterone treatment on adult penile size why sex reversal is not indicated. Journal of Pediatrics 1999134 579-583. (https://doi. org/10.1016/S0022-3476(99)70244-1)

7 Lee PA \& Houk CP. Outcome studies among men with micropenis. Journal of Pediatric Endocrinology and Metabolism 200417 1043-1053. (https://doi.org/10.1515/JPEM.2004.17.2.133)

8 Husmann DA \& Cain MP. Microphallus: eventual phallic size is dependent on the timing of androgen administration. Journal of Urology 1994152 734-739. (https://doi.org/10.1016/S00225347(17)32694-0)

9 Laron Z \& Sarel R. Penis and testicular size in patients with growth hormone insufficency. Acta Endocrinologica 197063 625-633. (https://doi.org/10.1530/acta.0.0630625)

10 Lee SW, Kim SH, Kim JY \& Lee Y. The effect of growth hormone on fibroblast proliferation and keratinocyte migration. Journal of Plastic, Reconstructive and Aesthetic Surgery 201063 e364-e369. (https://doi. org/10.1016/j.bjps.2009.10.027)

11 Felix-Patricio B, De Souza DB, Gregorio BM, Costa WS \& Sampaio FJ. How to quantify penile corpus cavernosum structures with histomorphometry: comparison of two methods. BioMed Research International 20152015 832156. (https://doi.org/10.1155/2015/832156)
12 Lee H, Jin MH, Kang HJ, Hong CH, Bang WJ, Park KK \& Han SW. Timing of prepubertal androgen administration may have different effects on future fertility as well as penile size in normal male rats. Urology 201075 979-984. (https://doi.org/10.1016/j. urology.2009.08.064)

13 Levy JB, Seay TM, Tindall DJ \& Husmann DA. The effects of androgen administration on phallic androgen receptor expression. Journal of Urology 1996156 775-779. (https://doi.org/10.1016/S00225347(01)65812-9)

14 Levy JB \& Husmann DA. Micropenis secondary to growth hormone deficiency: does treatment with growth hormone alone result in adequate penile growth? Journal of Urology 1996156 214-216. (https://doi.org/10.1016/S0022-5347(01)66003-8)

15 Sircili MH, e Silva FA, Costa EM, Brito VN, Arnhold IJ, Denes FT, Inacio M \& de Mendonca BB. Long-term surgical outcome of masculinizing genitoplasty in large cohort of patients with disorders of sex development. Journal of Urology $2010 \mathbf{1 8 4} 1122-1127$. (https:// doi.org/10.1016/j.juro.2010.05.022)

16 Traish A \& Kim N. The physiological role of androgens in penile erection: regulation of corpus cavernosum structure and function. Journal of Sexual Medicine 20052 759-770. (https://doi.org/10.1111/ j.1743-6109.2005.00094.x)

17 Okumu LA, Braden TD, Vail K, Simon L \& Goyal HO. Low androgen induced penile maldevelopment involves altered gene expression of biomarkers of smooth muscle differentiation and a key enzyme regulating cavernous smooth muscle cell tone. Journal of Urology 2014 192 267-273. (https://doi.org/10.1016/j.juro.2013.11.101)

18 Albin AK, Ankarberg-Lindgren C, Tuvemo T, Jonsson B, AlbertssonWikland K \& Ritzen EM. Does growth hormone treatment influence pubertal development in short children? Hormone Research in Paediatrics 201176 262-272. (https://doi.org/10.1159/000329743)

19 Lischka A. Investigation of peripheral androgen resistance in genital hypoplasia associated with congenital growth hormone deficiency. Andrologia 1987 19 97-102. (https://doi. org/10.1111/j.1439-0272.1987.tb01868.x)

20 Kapitola J, Kubickova J \& Andrle J. Blood flow and mineral content of the tibia of female and male rats: changes following castration and/or administration of estradiol or testosterone. Bone 199516 69-72. (https://doi.org/10.1016/8756-3282(95)80013-G)

Received in final form 4 September 2018

Accepted 10 September 2018

Accepted Preprint published online 10 September 2018

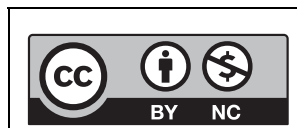

This work is licensed under a Creative Commons Attribution-NonCommercial 4.0 International License. 